\title{
A comparison of alternative c-conjugate dual problems in infinite convex optimization
}

\author{
M.D. FAJARDO ${ }^{1}$, J. VIDAL \\ Department of Mathematics \\ University of Alicante, 03080 Alicante, Spain
}

\begin{abstract}
In this work we obtain a Fenchel-Lagrange dual problem for an infinite dimensional optimization primal one, via perturbational approach and using a conjugation scheme called c-conjugation instead of classical Fenchel conjugation. This scheme is based on the generalized convex conjugation theory. We analyze some inequalities between the optimal values of Fenchel, Lagrange and Fenchel-Lagrange dual problems and we establish sufficient conditions under which they are equal. Examples where such inequalities are strictly fulfilled are provided. Finally, we study the relations between the optimal solutions and the solvability of the three mentioned dual problems.
\end{abstract}

Keywords: Evenly convex function, generalized convex conjugation, Fenchel, Lagrange and Fenchel-Lagrange dual problems.

Mathematics Subject Classification (2010): 52A20, 26B25, 90C25, 49N15.

${ }^{1}$ Corresponding author. E-mail adresses: md.fajardo@ua.es and jose.vidal@ua.es.

* This research was partially supported by MINECO of Spain and ERDF of EU, Grant MTM2014-59179C2-1-P and by Consellería de la Educación de la Generalitat Valenciana, Spain, Pre-doc Program Vali + d, DOCV 6791/07.06.2012, Grant ACIF-2013-156.

\section{Introduction}

Let us consider $X$ a separated locally convex space and the general optimization problem

$$
\begin{array}{lll}
(G P) & \operatorname{Inf} & F(x) \\
& \text { s.t. } & x \in X,
\end{array}
$$

where $F: X \rightarrow \overline{\mathbb{R}}:=\mathbb{R} \cup\{ \pm \infty\}$ is a proper function. Applying the perturbational approach introduced first by Ekeland and Teman in [6], whose key is to use a perturbation function $\Phi: X \times \Theta \rightarrow \overline{\mathbb{R}}$, such that $\Phi(x, 0)=F(x)$, for all $x \in X$, being $\Theta$ the space of perturbation variables, a dual problem to $(G P)$ can be built as follows

$$
\begin{aligned}
& (G D) \text { Sup }-\Phi^{*}\left(0, z^{*}\right) \\
& \text { s.t. } z^{*} \in \Theta^{*} \text {, }
\end{aligned}
$$

where $X^{*}, \Theta^{*}$ are the topological dual spaces of $X$ and $\Theta$, respectively, and $\Phi^{*}: X^{*} \times \Theta^{*} \rightarrow \overline{\mathbb{R}}$ is the Fenchel conjugate function of $\Phi$. If we denote by $v(G P)$ and $v(G D)$ the optimal values of the primal and the dual problems, respectively, a direct consequence of Fenchel-Young inequality is $v(G P) \geq v(G D)$, situation known as weak duality. The difference between the optimal values of the primal problem and the dual one is called duality gap, and it is said that there exists strong duality when there is no duality gap and the dual problem is solvable. Sufficient conditions for strong duality are called regularity conditions. This well-known 
framework will be named the classical setting throughout this work.

Combining the perturbation functions used to get the celebrated Fenchel and Lagrange dual problems, it is possible to build a new dual one, which is called Fenchel-Lagrange dual problem. This new dual problem was coined by Bot and Wanka in [23] in a finite dimensional setting. Moreover, they studied the inequality relations between the optimal values of Fenchel-Lagrange dual problem and Fenchel and Lagrange dual ones, establishing sufficient conditions for equality. The issue of getting regularity conditions for strong Fenchel-Lagrange duality in the classical context was addressed in [3,4] and [1]. In [2], applications of this new dual approach were given to solve some problems considered by Scott and Jefferson in [19] whereas in [20, 21, 22], some recent research about Fenchel and Fenchel-Lagrange dual problems by using the standard conjugation scheme can also be found.

In this paper we will deal with another conjugation scheme to build Fenchel-Lagrange dual problem in general separated locally convex spaces. This pattern, which is inspired on a survey done by Martínez-Legaz in [14] where generalized convex duality theory is applied to quasiconvex programming, is called c-conjugation scheme and it was developed in [15]. In the same way than in the classical context, convexity and lower semicontinuity of the perturbation function are required in most of the regularity conditions (see [1]), the use of the c-conjugation scheme instead of classical Fenchel one is associated with the evenly convexity of such a function. As a matter of fact, according to [18], evenly convex sets (functions) are a generalization of closed and convex sets (functions).

Due to Fenchel [9], a subset $C \subseteq X$ is said to be evenly convex, e-convex in brief, if it is the intersection of an arbitrary family, possibly empty, of open halfspaces. The properties of these sets have been applied to study the solvability of semi-infinite linear systems containing infinitely many strict inequalities in [11], and some important properties in terms of their sections and projections are given in [13]. According to [18], a function $f: X \rightarrow \overline{\mathbb{R}}$ is said to be e-convex if its epigraph is e-convex in $X \times \mathbb{R}$. According to [15], the c-conjugation scheme is suitable for this class of functions in the sense that the double conjugate function equals the original one whenever it is proper and e-convex. This result, can be understood as the e-convex counterpart of the so-called Fenchel-Moreau theorem.

In the classical context, the structure of Fenchel-Lagrange dual problem is related to the one of Fenchel and Lagrange dual ones (see [23]). These two dual problems have been already adapted by using the c-conjugation scheme in [8] and [7], respectively, so it is natural to wonder what would happen with the insights of Fenchel-Lagrange dual problem using this more general conjugation scheme. In particular, we will study the structure of this dual problem, and what kind of relation exists between the optimal values, the optimal solutions and the solvability of Fenchel, Lagrange and Fenchel-Lagrange dual problems.

The layout of this work is as follows. In Section 2 we present all the preliminary results regarding e-convex sets and functions to make this paper self-contained. Section 3 is gathered to the establishment of Fenchel-Lagrange dual problem associated to a general primal one using the perturbational approach and the c-conjugation scheme. Section 4 is devoted to make a comparison of the optimal values of Fenchel, Lagrange and Fenchel-Lagrange dual problems obtained by this approach. In this section we also provide the counterpart sufficient 
conditions under which the optimal value of Fenchel-Lagrange dual problem is equal, on the one hand to the one of Fenchel dual problem and, on the other hand, to the optimal value of Lagrange dual problem. Finally, in Section 5 we study the relations between the optimal solutions of these three dual problems and their solvability.

\section{Preliminaries}

Let $X$ be a separated locally convex space, lcs in brief, equipped with the $\sigma\left(X, X^{*}\right)$ topology induced by $X^{*}$, its continuous dual space which will be endowed with the $\sigma\left(X^{*}, X\right)$ topology. The notation $\left\langle x, x^{*}\right\rangle$ stands for the value at $x \in X$ of the continuous linear functional $x^{*} \in X^{*}$. For a set $D \subseteq X$ we will denote its convex hull, its closure and its interior by $\operatorname{conv} D, \operatorname{cl} D$ and int $D$, respectively. Moreover, if $D \neq \emptyset$, the indicator function $\delta_{D}: X \rightarrow \overline{\mathbb{R}}$ is defined by

$$
\delta_{D}(x)= \begin{cases}0 & \text { if } x \in D \\ +\infty & \text { otherwise }\end{cases}
$$

According to [5], a set $C \subseteq X$ is evenly convex, e-convex in short, if for every point $x_{0} \notin C$, there exists $x^{*} \in X^{*}$ such that $\left\langle x-x_{0}, x^{*}\right\rangle<0$, for all $x \in C$. Furthermore, for a set $C \subseteq X$, the e-convex hull of $C$, econv $C$, is the smallest e-convex set in $X$ containing $C$. This operator is well defined because the class of e-convex sets is closed under arbitrary intersections. Moreover, since $X$ is assumed to be a separated lcs, $X^{*} \neq \emptyset$ and, as a consequence of HahnBanach theorem, it holds that $X$ is e-convex and every closed or open convex set is e-convex as well.

On the other hand, for all $C \subseteq X$, it always holds $C \subseteq$ conv $C \subseteq$ econv $C \subseteq \operatorname{cl} C$ and, if $C_{1} \subseteq \mathbb{R}^{n}$ and $C_{2} \subseteq \mathbb{R}^{m}$ are e-convex in $\mathbb{R}^{n}$ and $\mathbb{R}^{m}$, respectively, then $C_{1} \times C_{2}$ is e-convex in $\mathbb{R}^{n+m}$. This property was shown by Goberna et al. in [10], but it can be extended to the infinite dimensional case with no extra effort.

Considering now a function $f: X \rightarrow \overline{\mathbb{R}}$, we denote by

$$
\operatorname{dom} f=\{x \in X: f(x)<+\infty\}
$$

the effective domain of $f$ and by

$$
\text { epi } f=\{(x, r) \in X \times \mathbb{R}: f(x) \leq r\}
$$

its epigraph. We say that $f$ is proper if epi $f$ does not contain vertical lines, or equivalently, if $\operatorname{dom} f \neq \emptyset$. Given $f, g: X \rightarrow \overline{\mathbb{R}}$ two proper convex functions, the infimal convolution of $f$ and $g$ is the function $f \square g: X \rightarrow \overline{\mathbb{R}}$ defined as

$$
(f \square g)(x):=\inf _{x_{1}+x_{2}=x}\left\{f\left(x_{1}\right)+g\left(x_{2}\right)\right\},
$$

and it is said to be exact at a point $a \in X$ if $(f \square g)(a)=f(x)+g(a-x)$ for some $x \in X$. The infimal convolution is exact if it is exact at any $x \in X$. By cl $f$ we denote the lower semicontinuous hull of $f$, which is the function whose epigraph equals cl(epi $f$ ). A function $f$ 
is lower semicontinuous, lsc in brief, if for all $x \in X, f(x)=\operatorname{cl} f(x)$. Clearly, any lsc convex function is e-convex, but the converse does not hold in general as we can see in the following example.

Example 2.1. Let $f: \mathbb{R} \rightarrow \overline{\mathbb{R}}$ be the function defined as

$$
f(x)= \begin{cases}x & \text { if } x>0 \\ +\infty & \text { otherwise. }\end{cases}
$$

Clearly, epi $f=\left\{(x, y) \in \mathbb{R}^{2} \mid x>0, y \geq x\right\}$ is e-convex in $\mathbb{R} \times \mathbb{R}$ since for every $x_{0} \notin$ epi $f$, there exists a non-trivial hyperplane whose intersection with epi $f$ is empty. However, it is not closed and, consequently, $f$ is not lsc.

The e-convex hull of a function $f: X \rightarrow \overline{\mathbb{R}}$, econv $f$, is defined as the function such that

$$
\operatorname{epi}(\operatorname{econv} f)=\operatorname{econv}(\operatorname{epi} f)
$$

or, equivalently, the largest e-convex minorant of $f$. Based on the generalized convex conjugation theory introduced by Moreau [16], a suitable conjugation scheme for e-convex functions is provided in [15]. Let us consider the space $W:=X^{*} \times X^{*} \times \mathbb{R}$ with the coupling functions $c: X \times W \rightarrow \overline{\mathbb{R}}$ and $c^{\prime}: W \times X \rightarrow \overline{\mathbb{R}}$ given by

$$
c\left(x,\left(x^{*}, y^{*}, \alpha\right)\right)=c^{\prime}\left(\left(x^{*}, y^{*}, \alpha\right), x\right):= \begin{cases}\left\langle x, x^{*}\right\rangle & \text { if }\left\langle x, y^{*}\right\rangle<\alpha, \\ +\infty & \text { otherwise. }\end{cases}
$$

Given two functions $f: X \rightarrow \overline{\mathbb{R}}$ and $g: W \rightarrow \overline{\mathbb{R}}$, the c-conjugate of $f$ and the $c^{\prime}$-conjugate of $g$ are defined as the functions $f^{c}: W \rightarrow \overline{\mathbb{R}}$ and $g: W \rightarrow \overline{\mathbb{R}}$, such that

$$
\begin{aligned}
f^{c}\left(x^{*}, y^{*}, \alpha\right) & :=\sup _{x \in X}\left\{c\left(x,\left(x^{*}, y^{*}, \alpha\right)\right)-f(x)\right\}, \\
g^{c^{\prime}}(x) & :=\sup _{\left(x^{*}, y^{*}, \alpha\right) \in W}\left\{c^{\prime}\left(\left(x^{*}, y^{*}, \alpha\right), x\right)-g\left(x^{*}, y^{*}, \alpha\right)\right\},
\end{aligned}
$$

respectively, with the conventions $(+\infty)+(-\infty)=(-\infty)+(+\infty)=(+\infty)-(+\infty)=$ $(-\infty)-(-\infty)=-\infty$.

Next result can be understood as the counterpart of Fenchel-Moreau theorem for e-convex functions.

Theorem 2.2. [15, Cor. 41] A function $f: X \rightarrow \mathbb{R} \cup\{+\infty\}$ is $e$-convex if and only if $f^{c c^{\prime}}=f$.

To conclude this section, we introduce the optimization problem we will deal with in the sequel,

$$
\begin{array}{lll}
(P) & \text { Inf } & f(x) \\
& \text { s.t. } & x \in A,
\end{array}
$$


where the feasible set

$$
A:=\left\{x \in X \mid g_{t}(x) \leq 0, \forall t \in T\right\}
$$

is non-empty, $f, g_{t}: X \rightarrow \overline{\mathbb{R}}$ are proper functions, for all $t \in T$, and $T$ is an arbitrary (possibly infinite) index set. Defining $g: X \rightarrow \mathbb{R}^{T}$ the vector-valued function such that

$$
g(x)(t)=g_{t}(x)
$$

for all $x \in X$ and $t \in T$, the set $A$ can be expressed as follows

$$
\left\{x \in X \mid g(x) \in-\mathbb{R}_{+}^{T}\right\}
$$

Associated to the space $\mathbb{R}^{T}$ we introduce the so-called space of generalized finite sequences in $\mathbb{R}$, denoted by $\mathbb{R}^{(T)}$, whose elements, $\lambda=\left(\lambda_{t}\right)_{t \in T}$, vanish everywhere except on a finite subset of $T$. In our setting, $\mathbb{R}^{(T)}$ will be identified as the dual space of $\mathbb{R}^{T}$ considering as dual product

$$
\lambda b=\sum_{t \in T} \lambda_{t} \cdot b_{t}
$$

for all $\lambda \in \mathbb{R}^{(T)}$ and $b \in \mathbb{R}^{T}$.

Remark 2.3. It is worthwhile mentioning that the feasibility condition (3) in the primal problem (1) can be formulated in a more general way. To this aim, observe that one can replace the cone $\mathbb{R}_{+}^{T}$ by a general non-empty closed convex cone $S \subseteq \mathbb{R}^{T}$ which induces a partial order in the separated locally convex space $\mathbb{R}^{T}$.

\section{Fenchel-Lagrange dual problem}

In the classical context, the perturbational approach allows us to obtain some different dual problems for the primal one defined in (1). In particular, considering the following perturbation functions

$$
\begin{aligned}
\Phi_{F}(x, u) & := \begin{cases}f(x+u) & \text { if } x \in A, \\
+\infty & \text { otherwise, }\end{cases} \\
\Phi_{L}(x, b) & := \begin{cases}f(x) & \text { if } g_{t}(x) \leq b_{t}, \forall t \in T, \\
+\infty & \text { otherwise, }\end{cases} \\
\Phi_{F L}(x, y, b) & := \begin{cases}f(x+y) & \text { if } g_{t}(x) \leq b_{t}, \forall t \in T, \\
+\infty & \text { otherwise, }\end{cases}
\end{aligned}
$$

the classical version of Fenchel, Lagrange and Fenchel-Lagrange dual problems associated to the primal problem $(P)$ are

$$
\left(\bar{D}_{F}\right) \operatorname{Sup}_{u^{*} \in X^{*}}\left\{-f^{*}\left(u^{*}\right)-\delta_{A}^{*}\left(-u^{*}\right)\right\}, \quad\left(\bar{D}_{L}\right) \operatorname{Sup}_{\lambda \in \mathbb{R}_{+}^{(T)}}\left\{\inf _{x \in X}\{f(x)+\lambda g(x)\}\right\}
$$


and

$$
\left(\bar{D}_{F L}\right) \operatorname{Sup}_{\substack{u^{*} \in X^{*} \\ \lambda \in \mathbb{R}_{+}^{(T)}}}\left\{-f^{*}\left(u^{*}\right)-(\lambda g)^{*}\left(-u^{*}\right)\right\}
$$

In [8] and [7], the c-conjugation pattern was applied to the perturbation functions $\Phi_{F}$ and $\Phi_{L}$ obtaining the following Fenchel and Lagrange dual problems,

$$
\begin{aligned}
& \left(D_{F}\right) \operatorname{Sup}_{y^{*}, v^{*} \in X^{*}},\left\{-f^{c}\left(y^{*}, v^{*}, \alpha_{1}\right)-\delta_{A}^{c}\left(-y^{*},-v^{*}, \alpha_{2}\right)\right\}, \\
& \left(D_{L}\right) \operatorname{Sup}_{\lambda \in \mathbb{R}_{+}^{(T)}}\left\{\inf _{x \in X}\{f(x)+\lambda g(x)\}\right\} .
\end{aligned}
$$

Our challenge in this section is to get Fenchel-Lagrange dual problem applying the cconjugation scheme to the perturbation function $\Phi_{F L}$ defined in (4). To this aim, taking into account the vector-valued function defined in (2), the perturbation function $\Phi_{F L}$ can be expressed as follows

$$
\Phi_{F L}(x, y, b)= \begin{cases}f(x+y) & \text { if } g(x)-b \in-\mathbb{R}_{+}^{T} \\ +\infty & \text { otherwise }\end{cases}
$$

Now, let us define the space $Z:=X \times X \times \mathbb{R}^{T}$ and let us identify its dual space as $Z^{*}=X^{*} \times X^{*} \times \mathbb{R}^{(T)}$. The suitable coupling function to build the c-conjugate of $\Phi_{F L}$ is $c_{1}: Z \times Z^{*} \times Z^{*} \times \mathbb{R} \rightarrow \overline{\mathbb{R}}$, defined as

$c_{1}\left((x, y, b),\left(\left(x^{*}, y^{*}, \lambda\right),\left(u^{*}, v^{*}, \beta\right), \alpha\right)\right)= \begin{cases}\left\langle x, x^{*}\right\rangle+\left\langle y, y^{*}\right\rangle+\lambda b & \text { if }\left\langle x, u^{*}\right\rangle+\left\langle y, v^{*}\right\rangle+\beta b<\alpha, \\ +\infty & \text { otherwise }\end{cases}$

Then, the c-conjugate of $\Phi_{F L}$ is the function $\Phi_{F L}^{c_{1}}: Z^{*} \times Z^{*} \times \mathbb{R} \rightarrow \overline{\mathbb{R}}$ such that

$$
\Phi_{F L}^{c_{1}}\left(\left(x^{*}, y^{*}, \lambda\right),\left(u^{*}, v^{*}, \beta\right), \alpha\right)=\sup _{(x, y, b) \in Z}\left\{c_{1}\left((x, y, b),\left(\left(x^{*}, y^{*}, \lambda\right),\left(u^{*}, v^{*}, \beta\right), \alpha\right)\right)-\Phi_{F L}(x, y, b)\right\}
$$

So, if we fix $x^{*}=0, u^{*}=0, y=0$ and $b=0$, we have

$$
\Phi_{F L}^{c_{1}}\left(\left(0, y^{*}, \lambda\right),\left(0, v^{*}, \beta\right), \alpha\right) \geq c_{1}\left((x, 0,0),\left(\left(0, y^{*}, \lambda\right),\left(0, v^{*}, \beta\right), \alpha\right)\right)-\Phi_{F L}(x, 0,0)
$$

for all $y^{*}, v^{*} \in X^{*}, \lambda, \beta \in \mathbb{R}^{(T)}$ and $\alpha>0$. Since $c_{1}\left((x, 0,0),\left(\left(0, y^{*}, \lambda\right),\left(0, v^{*}, \beta\right), \alpha\right)\right)=0$ for every $x \in X, y^{*}, v^{*} \in X^{*}, \lambda, \beta \in \mathbb{R}^{(T)}$ and $\alpha>0$, it holds

$$
\Phi_{F L}(x, 0,0) \geq-\Phi_{F L}^{c_{1}}\left(\left(0, y^{*}, \lambda\right),\left(0, v^{*}, \beta\right), \alpha\right)
$$

for all $x \in X, y^{*}, v^{*} \in X^{*}, \lambda, \beta \in \mathbb{R}^{(T)}$ and $\alpha>0$, leading us to formulate the dual problem

$$
\begin{aligned}
\left(D_{F L}^{1}\right) \quad \operatorname{Sup} \quad & -\Phi_{F L}^{c_{1}}\left(\left(0, y^{*}, \lambda\right),\left(0, v^{*}, \beta\right), \alpha\right) \\
& \lambda, \beta \in \mathbb{R}^{(T)} \\
\text { s.t. } \quad & y^{*}, v^{*} \in X^{*} \\
& \alpha>0 .
\end{aligned}
$$


Denoting by $v(P)$ and $v\left(D_{F L}^{1}\right)$ the optimal values of problems $(P)$ and $\left(D_{F L}^{1}\right)$, respectively, it holds $v(P) \geq v\left(D_{F L}^{1}\right)$, i.e., weak duality is fulfilled. In this point, we introduce the infimum value function $q: X \times \mathbb{R}^{T} \rightarrow \overline{\mathbb{R}}$, given by

$$
q(y, b):=\inf _{x \in X} \Phi_{F L}(x, y, b)
$$

To build its c-conjugate, let us consider the coupling function $c_{2}: U \times U^{*} \times U^{*} \times \mathbb{R} \rightarrow \overline{\mathbb{R}}$,

$$
c_{2}\left((y, b),\left(\left(y^{*}, \lambda\right),\left(v^{*}, \beta\right), \alpha\right)\right)= \begin{cases}\left\langle y, y^{*}\right\rangle+\lambda b & \text { if }\left\langle y, v^{*}\right\rangle+\beta b<\alpha \\ +\infty & \text { otherwise }\end{cases}
$$

being $U:=X \times \mathbb{R}^{T}$ and identifying $U^{*}=X^{*} \times \mathbb{R}^{(T)}$. Whence, we have $q^{c_{2}}: U^{*} \times U^{*} \times \mathbb{R} \rightarrow \overline{\mathbb{R}}$ and this function is defined as

$$
\begin{aligned}
q^{c_{2}}\left(\left(y^{*}, \lambda\right),\left(\left(v^{*}, \beta\right), \alpha\right)\right) & =\sup _{(x, y, b) \in Z}\left\{c_{2}\left((y, b),\left(\left(y^{*}, \lambda\right),\left(v^{*}, \beta\right), \alpha\right)\right)-\Phi_{F L}(x, y, b)\right\} \\
& =\sup _{(x, y, b) \in Z}\left\{c_{1}\left((x, y, b),\left(\left(0, y^{*}, \lambda\right),\left(0, v^{*}, \beta\right), \alpha\right)\right)-\Phi_{F L}(x, y, b)\right\} \\
& =\Phi_{F L}^{c_{1}}\left(\left(0, y^{*}, \lambda\right),\left(0, v^{*}, \beta\right), \alpha\right) .
\end{aligned}
$$

Then, the dual problem (6) can be rewritten as follows

$$
\begin{aligned}
\left(D_{F L}^{1}\right) \operatorname{Sup} \quad-q^{c_{2}}\left(\left(y^{*}, \lambda\right),\left(v^{*}, \beta\right), \alpha\right) & \\
& \lambda, \beta \in \mathbb{R}^{(T)} \\
\text { s.t. } \quad & y^{*}, v^{*} \in X^{*} \\
& \alpha>0 .
\end{aligned}
$$

Applying the definition of c-conjugate and infimum value functions, it holds

$$
q^{c_{2}}\left(\left(y^{*}, \lambda\right),\left(v^{*}, \beta\right), \alpha\right)=\sup _{\substack{x+y \in \operatorname{dom} f, g(x)-b \in-\mathbb{R}_{+}^{T}}}\left\{c_{2}\left((y, b),\left(\left(y^{*}, \lambda\right),\left(v^{*}, \beta\right), \alpha\right)\right)-f(x+y)\right\}
$$

since, with no loss of generality, we can assume that the supremum is taken over dom $q$. Introducing the new variables $s:=b-g(x) \in \mathbb{R}_{+}^{T}$ and $r:=x+y \in X$, we get

$$
q^{c_{2}}\left(\left(y^{*}, \lambda\right),\left(v^{*}, \beta\right), \alpha\right)=\sup _{\substack{x \in X, r \in \operatorname{dom}_{s \in \mathbb{R}_{+}^{T}} f}}\left\{c_{2}\left((r-x, s+g(x)),\left(\left(y^{*}, \lambda\right),\left(v^{*}, \beta\right), \alpha\right)\right)-f(r)\right\}
$$

where the coupling function $c_{2}\left((r-x, s+g(x)),\left(\left(y^{*}, \lambda\right),\left(v^{*}, \beta\right), \alpha\right)\right)$ is equal to

$$
\begin{cases}\left\langle r, y^{*}\right\rangle+\left\langle x,-y^{*}\right\rangle+\lambda s+\lambda g(x) & \text { if }\left\langle r, v^{*}\right\rangle+\left\langle x,-v^{*}\right\rangle+\beta s+\beta g(x)<\alpha, \\ +\infty & \text { otherwise. }\end{cases}
$$


Now, we claim that

$$
\begin{aligned}
\sup _{\substack{\lambda, \beta \in \mathbb{R}^{(T)}, y^{*}, v^{*} \in X^{*}, \alpha>0}} & -q^{c_{2}}\left(\left(y^{*}, \lambda\right),\left(v^{*}, \beta\right), \alpha\right) \\
& \geq \sup _{\substack{\lambda \in-\mathbb{R}_{+}^{(T)}, y^{*}, v^{*} \in X^{*}, \alpha>0, \alpha_{1} \in \mathbb{R}}}\left\{-f^{c}\left(y^{*}, v^{*}, \alpha_{1}\right)-(-\lambda g)^{c}\left(-y^{*},-v^{*}, \alpha-\alpha_{1}\right)\right\} .
\end{aligned}
$$

If the supremum in the left hand side of (8) is equal to $+\infty$, there is nothing to prove. Let us suppose that this supremum is finite. Then, we can restrict to $\lambda, \beta \in \mathbb{R}^{(T)}, y^{*}, v^{*} \in X^{*}$ and $\alpha>0$ such that $q^{c_{2}}\left(\left(y^{*}, \lambda\right),\left(v^{*}, \beta\right), \alpha\right)<+\infty$, which can be rewritten in this case as

$$
q^{c_{2}}\left(\left(y^{*}, \lambda\right),\left(v^{*}, \beta\right), \alpha\right)=f^{*}\left(y^{*}\right)+(-\lambda g)^{*}\left(-y^{*}\right)+\sup _{s \in \mathbb{R}_{+}^{T}}\{\lambda s\}
$$

with the implicit condition

$$
\left\langle r, v^{*}\right\rangle+\left\langle x,-v^{*}\right\rangle+\beta s+\beta g(x)<\alpha
$$

holding for all $r \in \operatorname{dom} f, x \in \operatorname{dom}(\lambda g)$ and $s \in \mathbb{R}_{+}^{T}$. From the finiteness of (9), and from condition (10), we deduce that $\lambda, \beta \in-\mathbb{R}_{+}^{(T)}$ and, hence

$$
\sup _{\substack{\lambda, \beta \in \mathbb{R}^{(T)}, y^{*}, v^{*} \in X^{*}, \alpha>0}}-q^{c_{2}}\left(\left(y^{*}, \lambda\right),\left(v^{*}, \beta\right), \alpha\right)=\sup _{\substack{\lambda, \beta \in-\mathbb{R}_{+}^{(T)} \\ y^{*}, v^{*} \in X^{*}, \alpha>0}}-q^{c_{2}}\left(\left(y^{*}, \lambda\right),\left(v^{*}, \beta\right), \alpha\right) .
$$

Now, take $\lambda, \beta \in-\mathbb{R}_{+}^{(T)}, y^{*}, v^{*} \in X^{*}$ and $\alpha>0$ such that $q^{c_{2}}\left(\left(y^{*}, \lambda\right),\left(v^{*}, \beta\right), \alpha\right)<+\infty$, meaning, from (9), that

$$
q^{c_{2}}\left(\left(y^{*}, \lambda\right),\left(v^{*}, \beta\right), \alpha\right)=f^{*}\left(y^{*}\right)+(-\lambda g)^{*}\left(-y^{*}\right)
$$

with the implicit condition (10). If there exists $\alpha_{1} \in \mathbb{R}$ such that

$$
\begin{aligned}
\operatorname{dom} f & \subseteq\left\{r \in X \mid\left\langle r, v^{*}\right\rangle<\alpha_{1}\right\} \\
\operatorname{dom}(-\lambda g) & \subseteq\left\{x \in X \mid\left\langle x,-v^{*}\right\rangle<\alpha-\alpha_{1}\right\}
\end{aligned}
$$

then $f^{*}\left(y^{*}\right)=f^{c}\left(y^{*}, v^{*}, \alpha_{1}\right)$ and $(-\lambda g)^{*}\left(-y^{*}\right)=(-\lambda g)^{c}\left(-y^{*},-v^{*}, \alpha-\alpha_{1}\right)$, and substituting in (11), we get

$$
-q^{c_{2}}\left(\left(y^{*}, \lambda\right),\left(v^{*}, \beta\right), \alpha\right)=-f^{c}\left(y^{*}, v^{*}, \alpha_{1}\right)-(-\lambda g)^{c}\left(-y^{*},-v^{*}, \alpha-\alpha_{1}\right) .
$$

If no $\alpha_{1} \in \mathbb{R}$ verified (12), either $f^{c}\left(y^{*}, v^{*}, \alpha_{1}\right)=+\infty$ or $(-\lambda g)^{c}\left(-y^{*},-v^{*}, \alpha-\alpha_{1}\right)=+\infty$ clearly, and, in this case,

$$
-q^{c_{2}}\left(\left(y^{*}, \lambda\right),\left(v^{*}, \beta\right), \alpha\right) \geq-f^{c}\left(y^{*}, v^{*}, \alpha_{1}\right)-(-\lambda g)^{c}\left(-y^{*},-v^{*}, \alpha-\alpha_{1}\right),
$$


getting, in both cases, inequality (8). Finally, let us check what happens if the supremum in the left hand side of (8) equals $-\infty$, meaning that

$$
q^{c_{2}} \equiv+\infty
$$

To get (13), it is enough with showing that, at any point,

$$
f^{c}\left(y^{*}, v^{*}, \alpha_{1}\right)+(-\lambda g)^{c}\left(-y^{*},-v^{*}, \alpha-\alpha_{1}\right)=+\infty .
$$

Proceeding by contradiction, if $\bar{y}^{*}, \bar{v}^{*} \in X^{*}, \bar{\lambda} \in-\mathbb{R}_{+}^{(T)}, \bar{\alpha}>0$ and $\bar{\alpha}_{1} \in \mathbb{R}$ verified

$$
\begin{aligned}
f^{c}\left(\bar{y}^{*}, \bar{v}^{*}, \bar{\alpha}_{1}\right) & <+\infty \\
(-\bar{\lambda} g)^{c}\left(-\bar{y}^{*},-\bar{v}^{*}, \bar{\alpha}-\bar{\alpha}_{1}\right) & <+\infty
\end{aligned}
$$

we would have

$$
\begin{aligned}
\left\langle r, \bar{v}^{*}\right\rangle & <\bar{\alpha}_{1}, \text { for all } r \in \operatorname{dom} f \\
\left\langle x,-\bar{v}^{*}\right\rangle & <\bar{\alpha}-\bar{\alpha}_{1}, \text { for all } x \in \operatorname{dom}(-\bar{\lambda} g) .
\end{aligned}
$$

Hence, as direct consequences of formulae (14) and (15) we would get, for all $r \in \operatorname{dom} f$, for all $x \in \operatorname{dom}(-\bar{\lambda} g)$ and for all $s \in \mathbb{R}_{+}^{T}$,

$$
\begin{aligned}
f^{*}\left(\bar{y}^{*}\right)+(-\bar{\lambda} g)^{*}\left(-\bar{y}^{*}\right) & <+\infty, \\
\left\langle r, \bar{v}^{*}\right\rangle+\left\langle x,-\bar{v}^{*}\right\rangle+0_{T} \cdot s+0_{T} \cdot g(x) & <\bar{\alpha} .
\end{aligned}
$$

Consequently, we would obtain $q^{c_{2}}\left(\left(\bar{y}^{*}, \bar{\lambda}\right),\left(\bar{v}^{*}, 0_{T}\right), \bar{\alpha}\right)<+\infty$, contradicting (13).

Thus, introducing the new variable $\alpha_{2}:=\alpha-\alpha_{1}$ in (8) and taking into account that $\lambda \in-\mathbb{R}_{+}^{(T)}$, we obtain what, from the practical point of view, we have called Fenchel-Lagrange dual problem, $\left(D_{F L}\right)$, associated to the primal problem $(P)$ defined in $(1)$,

$$
\begin{aligned}
\left(D_{F L}\right) \quad \operatorname{Sup} & \left\{-f^{c}\left(y^{*}, v^{*}, \alpha_{1}\right)-(\lambda g)^{c}\left(-y^{*},-v^{*}, \alpha_{2}\right)\right\} \\
& \lambda \in \mathbb{R}_{+}^{(T)} \\
\text { s.t. } \quad & y^{*}, v^{*} \in X^{*} \\
& \alpha_{1}+\alpha_{2}>0
\end{aligned}
$$

\section{Relations between the optimal values of Fenchel, Lagrange and Fenchel-Lagrange dual problems}

We have just shown that, as it happened in the classical context, Fenchel-Lagrange dual problem is also a combination of the well-known Fenchel and Lagrange dual problems when we use the c-conjugation scheme. This fact lets us think the following question: if the dual problems are related, what would happen with their optimal values? In [23], Bot and Wanka answered this question working on finite dimensional spaces having a finite number of constraints and dealing with the classical Fenchel conjugation scheme. Now, we will extend 
those results to general locally convex spaces having an arbitrary number of inequalities as constraints and working with the c-conjugation pattern.

First of all, we will study the relationship between these three dual problems, establishing the main inequalities that they satisfy as well as some examples where the inequalities are strictly fulfilled.

Proposition 4.1. Let $\left(D_{L}\right)$ and $\left(D_{F L}\right)$ be the dual problems defined in (5) and (16), respectively. It always holds $v\left(D_{L}\right) \geq v\left(D_{F L}\right)$. Moreover, if one of the following conditions fulfils

i) $f, g_{t}: X \rightarrow \overline{\mathbb{R}}$ are convex, for all $t \in T$, and $\operatorname{int}($ epi $f) \neq \emptyset$,

ii) there exist $\bar{\alpha}>0$ and $\left(\bar{y}^{*}, \bar{v}^{*}, \bar{\alpha}_{1}, \bar{\alpha}_{2}, \bar{\lambda}\right) \in W \times \mathbb{R} \times \mathbb{R}_{+}^{(T)}$ such that $\bar{\alpha}_{1}+\bar{\alpha}_{2}=\bar{\alpha}$ and

$$
f^{c}\left(\bar{y}^{*}, \bar{v}^{*}, \bar{\alpha}_{1}\right)+(\bar{\lambda} g)^{c}\left(-\bar{y}^{*},-\bar{v}^{*}, \bar{\alpha}_{2}\right) \leq \inf _{\lambda \in \mathbb{R}_{+}^{(T)}}\left\{(f+\lambda g)^{c}(0,0, \bar{\alpha})\right\}
$$

then $v\left(D_{L}\right)=v\left(D_{F L}\right)$.

Proof. First, let us show that $v\left(D_{L}\right) \geq v\left(D_{F L}\right)$ in any case. Let $\lambda \in \mathbb{R}_{+}^{(T)}$ and $y^{*} \in X^{*}$ arbitrary. Then it follows

$$
\begin{aligned}
\inf _{x \in X}\{f(x)+\lambda g(x)\} & =\inf _{x \in X}\left\{f(x)+\left\langle x, y^{*}\right\rangle+\left\langle x,-y^{*}\right\rangle+\lambda g(x)\right\} \\
& \geq \inf _{x \in X}\left\{\left\langle x,-y^{*}\right\rangle+f(x)\right\}+\inf _{x \in X}\left\{\left\langle x, y^{*}\right\rangle+\lambda g(x)\right\} \\
& =-\sup _{x \in X}\left\{\left\langle x, y^{*}\right\rangle-f(x)\right\}-\sup _{x \in X}\left\{\left\langle x,-y^{*}\right\rangle-(\lambda g)(x)\right\} \\
& =-f^{*}\left(y^{*}\right)-(\lambda g)^{*}\left(-y^{*}\right) .
\end{aligned}
$$

Since $f^{*}\left(y^{*}\right) \leq f^{c}\left(y^{*}, v^{*}, \alpha_{1}\right)$ and $(\lambda g)^{*}\left(-y^{*}\right) \leq(\lambda g)^{c}\left(-y^{*},-v^{*}, \alpha-\alpha_{1}\right)$ for all $v^{*} \in X^{*}, \alpha_{1} \in \mathbb{R}$ and $\alpha>0$, it yields

$$
\inf _{x \in X}\{f(x)+\lambda g(x)\} \geq-f^{c}\left(y^{*}, v^{*}, \alpha_{1}\right)-(\lambda g)^{c}\left(-y^{*},-v^{*}, \alpha-\alpha_{1}\right) .
$$

Defining $\alpha_{2}:=\alpha-\alpha_{1}$, constraints $\alpha_{1} \in \mathbb{R}$ and $\alpha>0$ can be joined in $\alpha_{1}+\alpha_{2}>0$. Now, since $\lambda \in \mathbb{R}_{+}^{(T)}$ and $y^{*} \in X^{*}$ were taken arbitrarily, it holds

$$
\begin{aligned}
v\left(D_{L}\right)= & \sup _{\substack{\lambda \in \mathbb{R}_{+}^{(T)} \\
\inf _{x \in X}}}\{f(x)+\lambda g(x)\} \\
& \sup _{\substack{y^{*}, v^{*} \in X^{*}, \alpha_{1}+\alpha_{2}>0, \lambda \in \mathbb{R}_{+}^{(T)}}}\left\{-f^{c}\left(y^{*}, v^{*}, \alpha_{1}\right)-(\lambda g)^{c}\left(-y^{*},-v^{*}, \alpha_{2}\right)\right\}=v\left(D_{F L}\right) .
\end{aligned}
$$


Now, let us prove that, under $i), v\left(D_{L}\right)=v\left(D_{F L}\right)$. It is sufficient to see that, for all $\lambda \in \mathbb{R}_{+}^{(T)}$,

$$
\inf _{x \in X}\{f(x)+\lambda g(x)\}=\sup _{\substack{y^{*}, v^{*} \in X^{*}, \alpha_{1}+\alpha_{2}>0}}\left\{-f^{c}\left(y^{*}, v^{*}, \alpha_{1}\right)-(\lambda g)^{c}\left(-y^{*},-v^{*}, \alpha_{2}\right)\right\} .
$$

Take any $\lambda \in \mathbb{R}_{+}^{(T)}$. In the case $\inf _{x \in X}\{f(x)+\lambda g(x)\}=-\infty$, from (17), we get (19). Hence, let us suppose that $\gamma:=\inf _{x \in X}\{f(x)+\lambda g(x)\} \in \mathbb{R}$. Again from (17), we have

$$
\begin{aligned}
\gamma & \geq \sup _{\substack{y^{*}, v^{*} \in X^{*}, \alpha_{1}+\alpha_{2}>0}}\left\{-f^{c}\left(y^{*}, v^{*}, \alpha_{1}\right)-(\lambda g)^{c}\left(-y^{*},-v^{*}, \alpha_{2}\right)\right\} \\
& \geq \sup _{\substack{y^{*} \in X^{*}, \alpha_{1}>0, \alpha_{2}>0}}\left\{-f^{c}\left(y^{*}, 0, \alpha_{1}\right)-(\lambda g)^{c}\left(-y^{*}, 0, \alpha_{2}\right)\right\} \\
& =\sup _{y^{*} \in X^{*}}\left\{-f^{*}\left(y^{*}\right)-(\lambda g)^{*}\left(-y^{*}\right)\right\} .
\end{aligned}
$$

Following the same steps as in [23, Prop 2], but instead of using [17, Th. 11.1, Th. 11.3], using [24, Th. 1.1.3], we obtain that there exists $y_{0}^{*} \in X^{*}$ such that, from (20), it verifies

$$
\begin{aligned}
-f^{*}\left(y_{0}^{*}\right)-(\lambda g)^{*}\left(-y_{0}^{*}\right) & \geq \gamma \geq \sup _{y^{*} \in X^{*}}\left\{-f^{*}\left(y^{*}\right)-(\lambda g)^{*}\left(-y^{*}\right)\right\} \\
& \geq-f^{*}\left(y_{0}^{*}\right)-(\lambda g)^{*}\left(-y_{0}^{*}\right)
\end{aligned}
$$

and

$$
\gamma=\inf _{x \in X}\{f(x)+\lambda g(x)\}=\sup _{\substack{y^{*}, v^{*} \in X^{*}, \alpha_{1}+\alpha_{2}>0}}\left\{-f^{c}\left(y^{*}, v^{*}, \alpha_{1}\right)-(\lambda g)^{c}\left(-y^{*},-v^{*}, \alpha_{2}\right)\right\} .
$$

Hence (19) is fulfilled, and taking suprema over $\lambda \in \mathbb{R}_{+}^{(T)}$, we get $v\left(D_{L}\right)=v\left(D_{F L}\right)$.

To conclude the proof, let us check that under $i i)$, again $v\left(D_{L}\right)=v\left(D_{F L}\right)$. Suppose that there exist $\bar{\alpha}>0$ and $\left(\bar{y}^{*}, \bar{v}^{*}, \bar{\alpha}_{1}, \bar{\alpha}_{2}, \bar{\lambda}\right) \in W \times \mathbb{R} \times \mathbb{R}_{+}^{(T)}$ such that $\bar{\alpha}_{1}+\bar{\alpha}_{2}=\bar{\alpha}$ and

$$
f^{c}\left(\bar{y}^{*}, \bar{v}^{*}, \bar{\alpha}_{1}\right)+(\bar{\lambda} g)^{c}\left(-\bar{y}^{*},-\bar{v}^{*}, \bar{\alpha}_{2}\right) \leq \inf _{\lambda \in \mathbb{R}_{+}^{(T)}}\left\{(f+\lambda g)^{c}(0,0, \bar{\alpha})\right\} .
$$

Then, a straightforward combination of (16), (18) and (21) implies that $v\left(D_{L}\right)=v\left(D_{F L}\right)$.

Remark 4.2. We should observe that if $X$ is finite dimensional, the assumption int (epi $f) \neq \emptyset$ in Proposition $4.1 \mathrm{i}$ ) can be eliminated according to [17, Th. 6.2].

The following example shows that if the underlying space is infinite dimensional, condition $\operatorname{int}($ epi $f) \neq \emptyset$ is necessary in Proposition 4.1 . 
Example 4.3. From Example 3.1 in [12], let us take $X=l^{2}$ the infinite dimensional Hilbert space of square-summable sequences. Let $T$ be a singleton index set. Let us define the linear subspaces

$$
\begin{aligned}
& C=\left\{x \in l^{2} \mid x_{2 n-1}+x_{2 n}=0, \text { for all } n \in \mathbb{N}\right\}, \\
& D=\left\{x \in l^{2} \mid x_{2 n}+x_{2 n+1}=0, \text { for all } n \in \mathbb{N}\right\},
\end{aligned}
$$

which are, indeed, closed and convex sets, and the functions $f, g: l^{2} \rightarrow \overline{\mathbb{R}}$ such that

$$
f(x)= \begin{cases}x_{1} & \text { if } x \in D \\ +\infty & \text { otherwise }\end{cases}
$$

and $g=\delta_{C}$. These functions are proper convex and lsc on $l^{2}$ with $\operatorname{dom} f=D, \operatorname{dom} g=C$ and $C \cap D=\{0\}$. Since $l^{2}$ is metrizable, it is not difficult to see that int $D=\emptyset$ and, consequently, $\operatorname{int}($ epi $f)=\emptyset$. Now, let us compute the domain of $f^{c}$ and $(\lambda g)^{c}$,

$$
\begin{aligned}
f^{c}\left(y^{*}, v^{*}, \alpha_{1}\right) & =\sup _{x \in D}\left\{c\left(x,\left(y^{*}, v^{*}, \alpha_{1}\right)\right)-x_{1}\right\} \\
& = \begin{cases}0 & \text { if }\left(y^{*}, v^{*}, \alpha_{1}\right) \in\left\{e_{1}+D^{\perp}\right\} \times\{0\} \times \mathbb{R}_{++}, \\
+\infty & \text { otherwise, }\end{cases} \\
(\lambda g)^{c}\left(-y^{*},-v^{*}, \alpha_{2}\right) & =\sup _{x \in C}\left\{c\left(x,\left(-y^{*},-v^{*}, \alpha_{2}\right)\right)-(\lambda g)(x)\right\} \\
& = \begin{cases}0 & \text { if }\left(y^{*}, v^{*}, \alpha_{2}\right) \in\left\{-C^{\perp}\right\} \times\{0\} \times \mathbb{R}_{++}, \\
+\infty & \text { otherwise, }\end{cases}
\end{aligned}
$$

where the superscript $\left(^{\perp}\right)$ stands for the orthogonal complement. Then, for all $\lambda \in \mathbb{R}_{+}$,

$$
\begin{aligned}
\operatorname{dom} f^{c} & =\left\{e_{1}+D^{\perp}\right\} \times\{0\} \times \mathbb{R}_{++}, \\
\operatorname{dom}(\lambda g)^{c} & =\left\{-C^{\perp}\right\} \times\{0\} \times \mathbb{R}_{++} .
\end{aligned}
$$

In [12], it was shown that $\left\{e_{1}+D^{\perp}\right\} \cap\left\{-C^{\perp}\right\}=\emptyset$ so, from (22), we get

$$
\operatorname{dom} f^{c} \cap \operatorname{dom}(\lambda g)^{c}=\emptyset,
$$

for all $\lambda \in \mathbb{R}_{+}$. Now, let us calculate $v\left(D_{F L}\right)$ and $v\left(D_{L}\right)$. Because of (23), it follows directly that $v\left(D_{F L}\right)=-\infty$. Finally, since $\operatorname{dom}(\lambda g)=C$ for all $\lambda \in \mathbb{R}_{+}$,

$$
v\left(D_{L}\right)=\sup _{\lambda \in \mathbb{R}_{+}} \inf _{x \in C \cap D}\{f(x)+(\lambda g)(x)\}=0 .
$$

Next example shows that the convexity of the involved functions in the primal problem cannot be removed in Proposition 4.1. 
Example 4.4. Let us take $X=\mathbb{R}, T$ a singleton index set, $f(x)=-x^{2}$ and $g(x)=x^{2}$. Clearly, $A=\{0\}$, int(epi $f) \neq \emptyset$ and

$$
\begin{aligned}
v\left(D_{L}\right) & =\sup _{\lambda \geq 0}\left\{\inf _{x \in \mathbb{R}}\left\{-x^{2}+\lambda x^{2}\right\}\right\}=\sup _{\lambda \geq 0}\left\{\inf _{x \in \mathbb{R}}\left\{x^{2}(\lambda-1)\right\}\right\} \\
& =\sup _{\lambda \geq 1}\left\{\inf _{x \in \mathbb{R}}\left\{x^{2}(\lambda-1)\right\}\right\}=0 .
\end{aligned}
$$

On the other hand,

$$
v\left(D_{F L}\right)=\sup _{\substack{y^{*}, v^{*} \in \mathbb{R} \\ \alpha_{1}+\alpha_{2}>0, \lambda \geq 0}}\left\{-\sup _{x \in \mathbb{R}}\left\{c\left(x,\left(y^{*}, v^{*}, \alpha_{1}\right)\right)+x^{2}\right\}-\sup _{x \in \mathbb{R}}\left\{c\left(x,\left(-y^{*},-v^{*}, \alpha_{2}\right)\right)-\lambda x^{2}\right\}\right\} .
$$

It is clear that we can restrict ourselves to $v^{*}=0$ and $\alpha_{1}, \alpha_{2}>0$, and we get

$$
v\left(D_{F L}\right)=\sup _{\substack{y^{*} \in \mathbb{R} \\ \lambda \geq 0}}\left\{\inf _{x \in \mathbb{R}}\left\{-x y^{*}-x^{2}\right\}+\inf _{x \in \mathbb{R}}\left\{x y^{*}+\lambda x^{2}\right\}\right\}=-\infty
$$

Proposition 4.5. Let $\left(D_{F}\right)$ and $\left(D_{F L}\right)$ be the dual problems defined in (5) and (16), respectively. It always holds $v\left(D_{F}\right) \geq v\left(D_{F L}\right)$.

Proof. Comparing problems $\left(D_{F}\right)$ with $\left(D_{F L}\right)$, it is easy to observe that we have to link $\delta_{A}(\cdot)$ with $(\lambda g)^{c}(\cdot)$, for $\lambda \in \mathbb{R}_{+}^{(T)}$. Take any $\left(y^{*}, v^{*}, \alpha_{2}\right) \in W$, then

$$
\delta_{A}^{c}\left(-y^{*},-v^{*}, \alpha_{2}\right)=\sup _{x \in X}\left\{c\left(x,\left(-y^{*},-v^{*}, \alpha_{2}\right)\right)-\delta_{A}(x)\right\} .
$$

Since $\lambda \in \mathbb{R}_{+}^{(T)}, \lambda g(x) \leq 0$ for every $x \in A$, and $\lambda g(x) \leq \delta_{A}(x)$ for each $x \in X$, so

$$
\delta_{A}^{c}\left(-y^{*},-v^{*}, \alpha_{2}\right) \leq \sup _{x \in X}\left\{c\left(x,\left(-y^{*},-v^{*}, \alpha_{2}\right)\right)-\lambda g(x)\right\}=(\lambda g)^{c}\left(-y^{*},-v^{*}, \alpha_{2}\right) .
$$

Then

$$
-f^{c}\left(y^{*}, v^{*}, \alpha_{1}\right)-\delta_{A}^{c}\left(-y^{*},-v^{*}, \alpha_{2}\right) \geq-f^{c}\left(y^{*}, v^{*}, \alpha_{1}\right)-(\lambda g)^{c}\left(-y^{*},-v^{*}, \alpha_{2}\right),
$$

for every $\lambda \in \mathbb{R}_{+}^{(T)}$ and $\alpha_{1} \in \mathbb{R}$ satisfying $\alpha_{1}+\alpha_{2}>0$, which allows us to conclude

$$
\begin{aligned}
v\left(D_{F}\right) & =\sup _{\substack{y^{*}, v^{*} \in X^{*}, \alpha_{1}+\alpha_{2}>0,}}\left\{-f^{c}\left(y^{*}, v^{*}, \alpha_{1}\right)-\delta_{A}^{c}\left(-y^{*},-v^{*}, \alpha_{2}\right)\right\} \\
\geq & \sup _{\substack{y^{*}, v^{*} \in X^{*}, \alpha_{1}+\alpha_{2}>0 \\
\lambda \in \mathbb{R}_{+}^{(T)}}}\left\{-f^{c}\left(y^{*}, v^{*}, \alpha_{1}\right)-(\lambda g)^{c}\left(-y^{*},-v^{*}, \alpha_{2}\right)\right\}=v\left(D_{F L}\right) .
\end{aligned}
$$


Now, the purpose is to find a condition under which Proposition 4.5 reads with equality. Let us consider the e-convex cone constraint qualification stated in Remark 4.1 and Definition 4.1 from [7], which is defined as

$$
\text { (ECCQ) } \quad \operatorname{epi} \delta_{A}^{c}=\bigcup_{\lambda \in \mathbb{R}_{+}^{(T)}} \operatorname{epi}(\lambda g)^{c}
$$

being $A \subseteq X$ a non-empty e-convex set, $g_{t}: X \rightarrow \overline{\mathbb{R}}$ proper and e-convex, for all $t \in T$, with $T$ an arbitrary index set.

Proposition 4.6. If (ECCQ) holds, then $v\left(D_{F}\right)=v\left(D_{F L}\right)$.

Proof. If $\delta_{A}^{c}\left(-y^{*},-v^{*}, \alpha_{2}\right)=+\infty$, for all $\left(y^{*}, v^{*}, \alpha_{2}\right) \in W$, we would obtain $v\left(D_{F}\right)=-\infty$ and, by Proposition 4.5, $v\left(D_{F}\right)=v\left(D_{F L}\right)$.

Now, let us assume that there exists at least a point $\left(y^{*}, v^{*}, \alpha_{2}\right) \in W$ where $\delta_{A}^{c}\left(-y^{*},-v^{*}, \alpha_{2}\right)$ is finite. In this case

$$
\beta:=-\delta_{A}^{c}\left(-y^{*},-v^{*}, \alpha_{2}\right)=\inf _{x \in A}\left\{-c\left(x,\left(-y^{*},-v^{*}, \alpha_{2}\right)\right)\right\} \in \mathbb{R}
$$

meaning that $\left\langle x,-v^{*}\right\rangle<\alpha_{2}$, for all $x \in A$, and

$$
\beta=\inf _{x \in A}\left\{-\left\langle x,-y^{*}\right\rangle\right\}=\inf _{x \in A}\left\{\left\langle x, y^{*}\right\rangle\right\}
$$

Due to the set $A \subseteq\left\{x \in X \mid\left\langle x,-v^{*}\right\rangle<\alpha_{2}\right\}$, we obtain that $\left\langle x, y^{*}\right\rangle=c\left(x,\left(y^{*},-v^{*}, \alpha_{2}\right)\right)$ for all $x \in A$ and, from (24),

$$
\beta=\inf _{x \in A}\left\{\left\langle x, y^{*}\right\rangle\right\}=\inf _{x \in A}\left\{c\left(x,\left(y^{*},-v^{*}, \alpha_{2}\right)\right)\right\}
$$

Applying Proposition 4.2 from [7], since $\sigma$ verifies (ECCQ) by hypothesis and $A$ is contained in the open half-space $\left\{x \in X \mid\left\langle x,-v^{*}\right\rangle<\alpha_{2}\right\}$, we get

$$
\beta=\inf _{x \in A}\left\{c\left(x,\left(y^{*},-v^{*}, \alpha_{2}\right)\right)\right\}=\max _{\lambda \in \mathbb{R}_{+}^{(T)}}\left\{\inf _{x \in X}\left\{c\left(x,\left(y^{*},-v^{*}, \alpha_{2}\right)\right)+\lambda g(x)\right\}\right\}
$$

and there exists a solution of (25), let us say $\bar{\lambda} \in \mathbb{R}_{+}^{(T)}$, such that

$$
\begin{aligned}
\beta & =\inf _{x \in A}\left\{c\left(x,\left(y^{*},-v^{*}, \alpha_{2}\right)\right)\right\}=\inf _{x \in \operatorname{dom} \bar{\lambda} g}\left\{-c\left(x,\left(-y^{*},-v^{*}, \alpha_{2}\right)\right)+\bar{\lambda} g(x)\right\} \\
& =-\sup _{x \in \operatorname{dom} \bar{\lambda} g}\left\{c\left(x,\left(-y^{*},-v^{*}, \alpha_{2}\right)\right)-\bar{\lambda} g(x)\right\}=-\sup _{x \in X}\left\{c\left(x,\left(-y^{*},-v^{*}, \alpha_{2}\right)\right)-\bar{\lambda} g(x)\right\} \\
& =-(\bar{\lambda} g)^{c}\left(-y^{*},-v^{*}, \alpha_{2}\right) .
\end{aligned}
$$

Then

$$
\beta=-\delta_{A}^{c}\left(-y^{*},-v^{*}, \alpha_{2}\right)=-(\bar{\lambda} g)^{c}\left(-y^{*},-v^{*}, \alpha_{2}\right)
$$


Adding $-f^{c}\left(y^{*}, v^{*}, \alpha_{1}\right)$ in both sides of (26), with $\alpha_{1}+\alpha_{2}>0$, and taking suprema with $y^{*}, v^{*} \in X^{*}$ and $\alpha_{1}+\alpha_{2}>0$, we get

$$
\begin{aligned}
v\left(D_{F}\right) & =\sup _{\substack{y^{*}, v^{*} \in X^{*}, \alpha_{1}+\alpha_{2}>0}}\left\{-f^{c}\left(y^{*}, v^{*}, \alpha_{1}\right)-\delta_{A}^{c}\left(-y^{*},-v^{*}, \alpha_{2}\right)\right\} \\
& =\sup _{\substack{y^{*}, v^{*} \in X^{*}, \alpha_{1}+\alpha_{2}>0}}\left\{-f^{c}\left(y^{*}, v^{*}, \alpha_{1}\right)-(\bar{\lambda} g)^{c}\left(-y^{*},-v^{*}, \alpha_{2}\right)\right\} \\
\leq & \sup _{\substack{y^{*}, v^{*} \in X^{*}, \alpha_{1}+\alpha_{2}>0, \lambda \in \mathbb{R}_{+}^{(T)}}}\left\{-f^{c}\left(y^{*}, v^{*}, \alpha_{1}\right)-(\lambda g)^{c}\left(-y^{*},-v^{*}, \alpha_{2}\right)\right\}=v\left(D_{F L}\right) .
\end{aligned}
$$

Applying Proposition 4.5, we conclude $v\left(D_{F}\right)=v\left(D_{F L}\right)$.

We conclude this section with an example which shows that condition (ECCQ) is necessary in Proposition 4.6.

Example 4.7. Let us take $X=\mathbb{R}^{2}, T=\left[1,+\infty\left[\right.\right.$ and $f, g_{t}: \mathbb{R}^{2} \rightarrow \overline{\mathbb{R}}$ such that, for all $t \in T$, $g_{t}(x)=t^{-1} \cdot x_{1}-x_{2}$ and

$$
f(x)= \begin{cases}x_{2} & \text { if } x_{1} \leq 0, x_{2} \in \mathbb{R} \\ +\infty & \text { otherwise }\end{cases}
$$

Firstly, let us see that these functions are e-convex. Clearly $g_{t}$ is e-convex for all $t \in T$. A matter of computation shows that if

$$
H=\left\{\left(x_{1}, x_{2}, x_{3}\right) \in \mathbb{R}^{3} \mid x_{3} \geq x_{2}\right\},
$$

then epi $f=H \cap(\operatorname{dom} f \times \mathbb{R})$. This set is clearly convex and closed, so $f$ is a proper lsc function and, as a consequence, it is e-convex. It is immediate to see that the set $A$ is

$$
A=\left\{\left(x_{1}, x_{2}\right) \in \mathbb{R}^{2} \mid x_{2} \geq 0, x_{2} \geq x_{1}\right\} .
$$

Hence, since $A \cap \operatorname{dom} f=\left\{\left(x_{1}, x_{2}\right) \in \mathbb{R}^{2} \mid x_{1} \leq 0, x_{2} \geq 0\right\}$, a simple calculation shows that $v(P)=0$. Now, let us continue calculating the optimal value of Lagrange dual problem.

$$
\begin{aligned}
v\left(D_{L}\right) & =\sup _{\lambda \in \mathbb{R}_{+}^{(T)}} \inf _{x \in \operatorname{dom} f}\left\{x_{2}+\sum_{t \in \operatorname{supp} \lambda} \lambda_{t}\left(t^{-1} \cdot x_{1}-x_{2}\right)\right\} \\
& =\sup _{\lambda \in \mathbb{R}_{+}^{(T)}} \inf _{x \in \operatorname{dom} f}\left\{x_{2}\left(1-\sum_{t \in \operatorname{supp} \lambda} \lambda_{t}\right)+\sum_{t \in \operatorname{supp} \lambda} \lambda_{t} \cdot t^{-1} \cdot x_{1}\right\}=-\infty .
\end{aligned}
$$

Since the involved functions are convex and $X=\mathbb{R}^{2}$, from Proposition 4.1, we get that $v\left(D_{F L}\right)=v\left(D_{L}\right)=-\infty$. If we compute the optimal value of Fenchel dual problem, we have 


$$
v\left(D_{F}\right)=\sup _{\substack{y, v \in \mathbb{R}^{2}, \alpha_{1}+\alpha_{2}>0}}\left\{-f^{c}\left(y, v, \alpha_{1}\right)-\left(\delta_{A}\right)^{c}\left(-y,-v, \alpha_{2}\right)\right\}
$$

It is not difficult to see that, at least, one of these c-conjugate functions equals $+\infty$ whenever $v \neq 0_{2}$. Analyzing the trivial case where $v_{1}=v_{2}=0$ and $\alpha_{1}, \alpha_{2}>0$, we get that $f^{c}\left(y, v, \alpha_{1}\right)$ and $\left(\delta_{A}\right)^{c}\left(-y,-v, \alpha_{2}\right)$ are finite, and

$$
\begin{aligned}
v\left(D_{F}\right) & =\sup _{y \in \mathbb{R}^{2}}\left\{-\sup _{x \in \operatorname{dom} f}\left\{x_{1} y_{1}+x_{2} y_{2}-x_{2}\right\}-\sup _{x \in A}\left\{-x_{1} y_{1}-x_{2} y_{2}\right\}\right\} \\
& \geq-\sup _{x \in \operatorname{dom} f}\left\{x_{1} \cdot 0+x_{2} \cdot 1-x_{2}\right\}+\inf _{x \in A}\left\{x_{1} \cdot 0+x_{2} \cdot 1\right\}=\inf _{x \in A} x_{2}=0 .
\end{aligned}
$$

We have just shown $v\left(D_{F}\right) \geq 0$ and, by weak duality, $v\left(D_{F}\right) \leq 0$, so $v\left(D_{F}\right)=0$. To conclude this example, it remains to see that

$$
\operatorname{epi} \delta_{A}^{c} \nsubseteq \bigcup_{t \in T} \operatorname{epi}(\lambda g)^{c}
$$

Clearly, $((0,-1),(0,-1), 1,0) \in \operatorname{epi} \delta_{A}^{c}$. However, this element does not belong to any epi $(\bar{\lambda} g)^{c}$ with $\bar{\lambda} \in \mathbb{R}_{+}^{(T)}$ since this fact would imply the fulfilment of

$$
c\left(\left(x_{1}, x_{2}\right),((0,-1),(0,-1), 1)\right)-(\bar{\lambda} g)\left(x_{1}, x_{2}\right) \leq 0,
$$

for all $\left(x_{1}, x_{2}\right) \in \operatorname{dom} \bar{\lambda} g=\mathbb{R}^{2}$. This would be equivalent to $\left\langle\left(x_{1}, x_{2}\right),(0,-1)\right\rangle<1$, for all $\left(x_{1}, x_{2}\right) \in \operatorname{dom}(\lambda g)=\mathbb{R}^{2}$, which is not true. Therefore, (27) does not hold.

Remark 4.8. If the underlying space is finite dimensional and the involved functions are proper and convex, applying Propositions 4.1 and 4.5 and Remark 4.2, it always holds

$$
v\left(D_{L}\right)=v\left(D_{F L}\right) \leq v\left(D_{F}\right)
$$

We should also observe that without any assumption over the primal problem, $v\left(D_{L}\right)$ and $v\left(D_{F}\right)$ cannot be related, even in the finite case. In fact, from Example 4.7, we have just seen that $v\left(D_{F}\right)>v\left(D_{L}\right)=v\left(D_{F L}\right)$ and, from Example 4.4, $v\left(D_{L}\right)>v\left(D_{F}\right)=v\left(D_{F L}\right)$.

\section{Relations between the optimal solutions of Fenchel, Lagrange and Fenchel-Lagrange dual problems}

In this section we study sufficient conditions for the equality of the three optimal values of Fenchel, Lagrange and Fenchel-Lagrange dual problems and the relationships between their optimal solutions. Clearly, this equality does not mean solvability in any case. For this reason, it is worth studying conditions under which the solvability of one of these dual problems implies the solvability of the others. 
On the one hand, it is not difficult to see that, for all $\alpha>0$,

$$
v(P)=\inf _{x \in X}\left\{f(x)+\delta_{A}(x)\right\}=-\left(f+\delta_{A}\right)^{c}(0,0, \alpha) .
$$

On the other hand, Fenchel-Lagrange dual problem can be expressed as follows,

$$
v\left(D_{F L}\right)=\sup _{\lambda \in \mathbb{R}_{+}^{(T)}}\left\{-\inf _{\substack{y^{*}, v^{*} \in X^{*}, \alpha_{1}+\alpha_{2}>0}}\left\{f^{c}\left(y^{*}, v^{*}, \alpha_{1}\right)+(\lambda g)^{c}\left(-y^{*},-v^{*}, \alpha_{2}\right)\right\}\right\} .
$$

Naming $\alpha:=\alpha_{1}+\alpha_{2}$, it follows

$$
v\left(D_{F L}\right)=\sup _{\lambda \in \mathbb{R}_{+}^{(T)}}\left\{-\left(f^{c} \square(\lambda g)^{c}\right)(0,0, \alpha)\right\}=-\inf _{\lambda \in \mathbb{R}_{+}^{(T)}}\left\{\left(f^{c} \square(\lambda g)^{c}\right)(0,0, \alpha)\right\} .
$$

Since

$$
\inf _{\lambda \in \mathbb{R}_{+}^{(T)}}\left\{\left(f^{c} \square(\lambda g)^{c}\right)(0,0, \alpha)\right\} \leq\left(f^{c} \square(\lambda g)^{c}\right)(0,0, \alpha)
$$

for all $\alpha>0$ and $\lambda \in \mathbb{R}_{+}^{(T)}$, we get

$$
v\left(D_{F L}\right) \geq-\left(f^{c} \square(\lambda g)^{c}\right)(0,0, \alpha) .
$$

Applying weak duality and formulae (28) and (29), it holds

$$
-\left(f^{c} \square(\lambda g)^{c}\right)(0,0, \alpha) \leq v\left(D_{F L}\right) \leq v(P)=-\left(f+\delta_{A}\right)^{c}(0,0, \alpha),
$$

for all $\alpha>0$ and for all $\lambda \in \mathbb{R}_{+}^{(T)}$, obtaining, finally, that for all $\alpha>0$ and for all $\lambda \in \mathbb{R}_{+}^{(T)}$, it yields

$$
\left(f+\delta_{A}\right)^{c}(0,0, \alpha) \leq\left(f^{c} \square(\lambda g)^{c}\right)(0,0, \alpha) .
$$

Proposition 5.1. If there exist $\bar{\lambda} \in \mathbb{R}_{+}^{(T)}$ and $\bar{\alpha}>0$ such that

$$
\left(f+\delta_{A}\right)^{c}(0,0, \bar{\alpha})=\left(f^{c} \square(\bar{\lambda} g)^{c}\right)(0,0, \bar{\alpha})
$$

and the infimal convolution is exact at $(0,0, \bar{\alpha})$, then

$$
v(P)=v\left(D_{L}\right)=v\left(D_{F}\right)=v\left(D_{F L}\right) .
$$

Proof. Let $\bar{\lambda} \in \mathbb{R}_{+}^{(T)}$ and $\bar{\alpha}>0$ verifying (30). The infimal convolution is exact at $(0,0, \bar{\alpha})$, so there exist $\bar{y}^{*}, \bar{v}^{*} \in X^{*}, \bar{\alpha}_{1}+\bar{\alpha}_{2}=\bar{\alpha}$ such that

$$
\begin{aligned}
-\left(f^{c} \square(\bar{\lambda} g)^{c}\right)(0,0, \bar{\alpha}) & =-f^{c}\left(\bar{y}^{*}, \bar{v}^{*}, \bar{\alpha}_{1}\right)-(\bar{\lambda} g)^{c}\left(-\bar{y}^{*},-\bar{v}^{*}, \bar{\alpha}_{2}\right) \\
\leq & \sup _{\substack{y^{*}, v^{*} \in X^{*}, \alpha_{1}+\alpha_{2}>0, \lambda \in \mathbb{R}_{+}^{(T)}}}\left\{-f^{c}\left(y^{*}, v^{*}, \alpha_{1}\right)-(\lambda g)^{c}\left(-y^{*},-v^{*}, \alpha_{2}\right)\right\}=v\left(D_{F L}\right) .
\end{aligned}
$$


Hence, from (30) and (28) we have $v(P) \leq v\left(D_{F L}\right)$ and, by virtue of weak duality, it follows that $v(P)=v\left(D_{F L}\right)$. The rest of the proof follows from Propositions 4.1 and 4.5.

Remark 5.2. Condition (30) in Proposition 5.1 can be understood as the e-convex counterpart of the constraint qualification introduced in Definition 4.1 in the renewed paper [22].

Proposition 5.3. If $v(P)=v\left(D_{F L}\right)$ and $\left(y_{0}^{*}, v_{0}^{*}, \bar{\alpha}_{1}, \bar{\alpha}_{2}, \bar{\lambda}\right) \in W \times \mathbb{R} \times \mathbb{R}_{+}^{(T)}$ is an optimal solution of $\left(D_{F L}\right)$ with $\alpha_{1}+\alpha_{2}>0$, then $\bar{\lambda}$ is optimal to $\left(D_{L}\right),\left(y_{0}^{*}, v_{0}^{*}, \bar{\alpha}_{1}, \bar{\alpha}_{2}\right)$ is optimal to $\left(D_{F}\right)$ and

$$
v(P)=v\left(D_{L}\right)=v\left(D_{F}\right)=v\left(D_{F L}\right) .
$$

Proof. From Proposition 4.1 and 4.5 we already know that

$$
v\left(D_{F L}\right) \leq v\left(D_{L}\right) \quad \text { and } \quad v\left(D_{F L}\right) \leq v\left(D_{F}\right)
$$

so if $v(P)=v\left(D_{F L}\right)$ we get (31). Now, let us suppose that $v(P)=v\left(D_{F L}\right) \in \mathbb{R}$ because otherwise applying (32) the conclusion is trivial. If $\left(y_{0}^{*}, v_{0}^{*}, \bar{\alpha}_{1}, \bar{\alpha}_{2}, \bar{\lambda}\right) \in W \times \mathbb{R} \times \mathbb{R}_{+}^{(T)}$ is an optimal solution of $\left(D_{F L}\right)$ with $\alpha_{1}+\alpha_{2}>0$, let us see first that $\bar{\lambda} \in \mathbb{R}_{+}^{(T)}$ is optimal to $\left(D_{L}\right)$. Since

$$
v\left(D_{L}\right)=v\left(D_{F L}\right)=-f^{c}\left(y_{0}^{*}, v_{0}^{*}, \bar{\alpha}_{1}\right)-(\bar{\lambda} g)^{c}\left(-y_{0}^{*},-v_{0}^{*}, \bar{\alpha}_{2}\right),
$$

and

$$
\begin{aligned}
f^{c}\left(y_{0}^{*}, v_{0}^{*}, \bar{\alpha}_{1}\right)+f(x) & \geq c\left(x,\left(y_{0}^{*}, v_{0}^{*}, \bar{\alpha}_{1}\right)\right), \text { for all } x \in X, \\
(\bar{\lambda} g)^{c}\left(-y_{0}^{*},-v_{0}^{*}, \bar{\alpha}_{2}\right)+(\bar{\lambda} g)(x) & \geq c\left(x,\left(-y_{0}^{*},-v_{0}^{*}, \bar{\alpha}_{2}\right)\right), \text { for all } x \in X,
\end{aligned}
$$

applying that the coupling function is subadditive in the second component, it follows

$$
v\left(D_{L}\right)=v\left(D_{F L}\right)=-f^{c}\left(y_{0}^{*}, v_{0}^{*}, \bar{\alpha}_{1}\right)-(\bar{\lambda} g)^{c}\left(-y_{0}^{*},-v_{0}^{*}, \bar{\alpha}_{2}\right) \leq f(x)+(\bar{\lambda} g)(x),
$$

for all $x \in X$. Then

$$
v\left(D_{L}\right)=v\left(D_{F L}\right) \leq \inf _{x \in X}\{f(x)+(\bar{\lambda} g)(x)\} \leq \sup _{\lambda \in \mathbb{R}_{+}^{(T)}}\left\{\inf _{x \in X}\{f(x)+\lambda g(x)\}\right\}=v\left(D_{L}\right),
$$

and $\bar{\lambda}$ is optimal to $\left(D_{L}\right)$. Similarly, but dealing with $v\left(D_{F}\right)=v\left(D_{F L}\right)$, it can be shown that $\left(y_{0}^{*}, v_{0}^{*}, \bar{\alpha}_{1}, \bar{\alpha}_{2}\right)$ is optimal to $\left(D_{F}\right)$.

Corollary 5.4. If there exists strong Fenchel-Lagrange duality, there also exist Fenchel and Lagrange strong duality.

Proposition 5.5. If $v\left(D_{L}\right)=v\left(D_{F}\right)=v\left(D_{F L}\right)$ and either $\left(D_{L}\right)$ or $\left(D_{F}\right)$ are not solvable, then $\left(D_{F L}\right)$ is not solvable. 
Proof. Let us start supposing that $\left(D_{L}\right)$ is not solvable. Then, for all $\lambda \in \mathbb{R}_{+}^{(T)}$, we have

$$
v\left(D_{L}\right)>\inf _{x \in X}\{f(x)+\lambda g(x)\}
$$

Suppose that $\left(D_{F L}\right)$ is solvable, then there exist $\bar{\lambda} \in \mathbb{R}_{+}^{(T)}, \bar{y}^{*}, \bar{v}^{*} \in X^{*}$ and $\bar{\alpha}_{1}+\bar{\alpha}_{2}>0$ such that

$$
v\left(D_{F L}\right)=-f^{c}\left(\bar{y}^{*}, \bar{v}^{*}, \bar{\alpha}_{1}\right)-(\bar{\lambda} g)^{c}\left(-\bar{y}^{*},-\bar{v}^{*}, \bar{\alpha}_{2}\right) .
$$

Then, since we are considering $v\left(D_{L}\right)=v\left(D_{F L}\right)$, we have, in particular,

$$
-f^{c}\left(\bar{y}^{*}, \bar{v}^{*}, \bar{\alpha}_{1}\right)-(\bar{\lambda} g)^{c}\left(-\bar{y}^{*},-\bar{v}^{*}, \bar{\alpha}_{2}\right)>\inf _{x \in X}\{f(x)+\lambda g(x)\}
$$

On the other hand, following the same steps as in the proof of Proposition 5.3, we get

$$
-f^{c}\left(\bar{y}^{*}, \bar{v}^{*}, \bar{\alpha}_{1}\right)-(\bar{\lambda} g)^{c}\left(-\bar{y}^{*},-\bar{v}^{*}, \bar{\alpha}_{2}\right) \leq \inf _{x \in X}\{f(x)+\lambda g(x)\}
$$

which is a contradiction with (33).

Similarly, if $\left(D_{F L}\right)$ were solvable but $\left(D_{F}\right)$ not, we would have

$-f^{c}\left(\bar{y}^{*}, \bar{v}^{*}, \bar{\alpha}_{1}\right)-(\bar{\lambda} g)^{c}\left(-\bar{y}^{*},-\bar{v}^{*}, \bar{\alpha}_{2}\right)=v\left(D_{F L}\right)=v\left(D_{F}\right)>-f^{c}\left(\bar{y}^{*}, \bar{v}^{*}, \bar{\alpha}_{1}\right)-\delta_{A}^{c}\left(-\bar{y}^{*},-\bar{v}^{*}, \bar{\alpha}_{2}\right)$

which is a contradiction since $(\lambda g)^{c} \geq \delta_{A}^{c}$ for all $\lambda \in \mathbb{R}_{+}^{(T)}$.

We conclude our work showing that the converse statement in the above proposition does not hold in general.

Example 5.6. Let us take $X=\mathbb{R}, f=\delta_{[0,+\infty}\left[, g_{t}(x)=t x\right.$ for all $t \in T=[0,+\infty[$. As in Example 5.1 from [7], $A=]-\infty, 0]$. A simple calculation shows, on the one hand, that $v(P)=0$ and, on the other hand,

$$
v\left(D_{L}\right)=\sup _{\lambda \in \mathbb{R}_{+}^{(T)}}\left\{\inf _{x \geq 0}\{\lambda g(x)\}\right\}=\sup _{\lambda \in \mathbb{R}_{+}^{(T)}}\left\{\inf _{x \geq 0}\left\{\sum_{t \in \operatorname{supp} \lambda} \lambda_{t} \cdot t x\right\}\right\}=0
$$

so every $\lambda \in \mathbb{R}_{+}^{(T)}$ is an optimal solution of $\left(D_{L}\right)$. Since $f^{c}\left(y^{*}, v^{*}, \alpha_{1}\right)<+\infty$ if and only if $\left(y^{*}, v^{*}, \alpha_{1}\right) \in \mathbb{R}_{-} \times \mathbb{R}_{-} \times \mathbb{R}_{++}$and its value is 0 , and $\delta_{A}^{c}\left(-y^{*},-v^{*}, \alpha_{2}\right)<+\infty$ if and only if $\left(y^{*}, v^{*}, \alpha_{2}\right) \in \mathbb{R}_{-} \times \mathbb{R}_{-} \times \mathbb{R}_{++}$being its value, again, 0 , it follows that $v\left(D_{F}\right)=0$ and the set of solutions of $\left(D_{F}\right)$ is $\mathbb{R}_{-} \times \mathbb{R}_{-} \times \mathbb{R}_{++} \times \mathbb{R}_{++}$.

Now, taking in particular $\bar{y}^{*}=\bar{v}^{*}=0, \bar{\alpha}_{1}, \bar{\alpha}_{2}>0$ and

$$
\bar{\lambda}= \begin{cases}1 & \text { if } t=1 \\ 0 & \text { otherwise }\end{cases}
$$


which are optimal solutions of $\left(D_{F}\right)$ and $\left(D_{L}\right)$, respectively, we get that $f^{c}\left(0,0, \bar{\alpha}_{1}\right)=0$ but

$$
(\bar{\lambda} g)^{c}\left(0,0, \bar{\alpha}_{2}\right)=\sup _{x \in \mathbb{R}}\left\{\langle x, 0\rangle-\sum_{t \in \operatorname{supp} \bar{\lambda}} \bar{\lambda}_{t} \cdot t x\right\}=\sup _{x \in \mathbb{R}}\{-x\}=+\infty .
$$

Then, $\left(0,0, \bar{\alpha}_{1}, \bar{\alpha}_{2}, \bar{\lambda}\right)$ is not optimal to $\left(D_{F L}\right)$ since, according to Proposition 4.1,

$$
v\left(D_{F L}\right)=v\left(D_{L}\right)=0
$$

\section{Further thoughts}

This work motivates us to analyze in a future paper new regularity conditions for strong Fenchel-Lagrange duality when the c-conjugation scheme is applied and the involved functions, and the feasible set in the primal problem, are e-convex, fact that also implies the perturbation function $\Phi_{F L}$ to be e-convex. This line of research could be interesting because as it happens in $[8,7]$ with Fenchel and Lagrange dual problems, the regularity conditions might be expressed in terms of evenly convexity. Hence, using the structure of FenchelLagrange dual problem developed in Section 3, the e-convex counterpart of the regularity conditions for such a kind of duality in the classical context could be adapted into this more general framework.

\section{References}

[1] R.I. Bot. Conjugate Duality in Convex Optimization, volume 637. Lecture Notes in Economics and Mathematical Systems, Springer-Verlag, Berlin Heidelberg, 2010.

[2] R.I. Bot, S. Grad, and G. Wanka. Fenchel-Lagrange versus geometric duality in convex optimization. J. Optim. Theory and Appl., 129(1):33-54, 2006.

[3] R.I. Bot, S. Grad, and G. Wanka. New regularity conditions for Lagrange and FenchelLagrange duality in infinite dimensional spaces. Math. Ineq. \& Appl., 12(1):171-189, 2009 .

[4] R.I. Boţ, G. Kassay, and G. Wanka. Strong duality for general convex optimization problems. J. Optim. Theory and Appl., 127(1):45-70, 2005.

[5] A. Daniilidis and J.E. Martínez-Legaz. Characterizations of evenly convex sets and evenly quasiconvex functions. J. Math. Anal. and Appl., 273:58-66, 2002.

[6] I. Ekeland and R. Temam. Convex analysis and variational problems. North-Holland Publishing Company, Amsterdam, 1976.

[7] M.D. Fajardo, M.M.L. Rodríguez, and J. Vidal. Lagrange duality for evenly convex optimization problems. J. Optim. Theory and Appl., 168(1):109-128, 2016.

[8] M.D. Fajardo, J. Vicente-Pérez, and M.M.L. Rodríguez. Infimal convolution, csubdifferentiability and Fenchel duality in evenly convex optimization. TOP, 20(2):375$396,2012$. 
[9] W. Fenchel. A remark on convex sets and polarity. Comm. Sèm. Math. Univ. Lund (Medd. Lunds Algebra Univ. Math. Sem.), pages 82-89, 1952. Tome Supplémentaire.

[10] M.A. Goberna, V. Jornet, and M.M.L. Rodríguez. On linear systems containing strict inequalities. Linear Algebra Appl., 360:151-171, 2003.

[11] M.A. Goberna and M.M.L. Rodríguez. Analyzing linear systems containing strict inequalities via evenly convex hulls. Eur. J. Oper. Res., 169:1079-1095, 2006.

[12] M.S. Gowda and M. Teboulle. A comparison of constraint qualifications in infinitedimensional convex programming. SIAM J. control Optim., 28(4):925-935, 1990.

[13] V. Klee, E. Maluta, and C. Zanco. Basic properties of evenly convex sets. J. Convex Anal., 14(1):137-148, 2006.

[14] J.E. Martínez-Legaz. Quasiconvex duality theory by generalized conjugation methods. Optimization, 19:603-652, 1988.

[15] J.E. Martínez-Legaz and J. Vicente-Pérez. The e-support function of an e-convex set and conjugacy for e-convex functions. J. Math. Anal. and Appl., 376:602-612, 2011.

[16] J.J. Moreau. Inf-convolution, sous-additivité, convexité des fonctions numériques. $J$. Math. Pures Appl., 49:109-154, 1970.

[17] R.T. Rockafellar. Convex Analysis. Princeton University Press, Princeton, New Yersey, 1970 .

[18] M.M.L. Rodríguez and J. Vicente-Pérez. On evenly convex functions. J. Convex Anal., 18:721-736, 2011.

[19] C.H. Scott and T.R. Jefferson. Duality for Minmax Programs. J. Math. Anal. and Appl., 100:385-393, 1984.

[20] X.K. Sun. Regularity conditions characterizing Fenchel-Lagrange duality and Farkastype results in DC infinite programming. J. Math. Anal. and App., 414(2):590-611, 2014.

[21] X.K. Sun, S.J. Li, and D. Zhao. Duality and Farkas-type results for DC infinite programming with inequality constraints. Taiwanese Journal of Math., 17(4):1227-1244, 2013.

[22] X.K. Sun, X.J. Long, and J. Zeng. Constraints qualifications characterizing Fenchel duality in composed convex optimization. J. Nonlinear and Convex Anal., 17(2):325$347,2016$.

[23] G. Wanka and R.I. Bot. On the relations between different dual problems in convex mathematical programming. Springer-Verlag, Heidelberg, Germany, 2002. P. Chamoni, R. Leisten, A. Martin, J. Minnemann and H. Stadtler.

[24] C. Zălinescu. Convex analysis in general vector spaces. World Scientific, New Jersey, 2002. 